PoS $\quad$ PROCEEDINGS

\title{
Experimental Status of Rare Decays in Charged Leptons and Light Mesons
}

\author{
Yoshitaka Kuno* \\ Department of Physics, Osaka Unviersity \\ E-mail: kunodphys.sci.osaka-u.ac.ip
}

We would like to present the experimental status of rare decays in charged leptons and light mesons, with the emphasis of the physics importance of rare decays and the development of high intensity and luminosity accelerators and particle sources.

36th International Conference on High Energy Physics,

July 4-11, 2012

Melbourne, Australia

${ }^{*}$ Speaker. 


\section{Why Rare Decays ?}

After the discovery of the Higgs boson at the LHC, we have a complete set of elementary particles in the Standard Model (SM). However, there are many parameters undetermined in the SM and many open issues. Therefore, we consider the SM is incomplete, being an only lowenergy approximation of the more complete theory. To get hints on the more complete theory at higher energy scale, we have to find new particles or new physics phenomena which the SM cannot expect.

To explore new physics beyond the SM, there are three identified frontiers in particle physics. They are the energy frontier, the cosmic frontier, and the intensity frontier. In the intensity frontier, intense beams are used to observe rare processes and study the particle properties to probe physics beyond the SM. And this is the frontier area which I am going to mention in this paper.

There are many rare decay processes in charged leptons and light mesons. Given the fact that new physics effects are not large, we might group rare decay processes into the three categories. One is a group of rare decay processes in which the SM contribution dominates, the second is those where the SM contribution is highly suppressed, and the third is those where the SM contribution is forbidden. Suppose the new physics contribution is given by $\mathrm{O}(\varepsilon)$, the second group has a new physics contribution in the order of $\mathrm{O}(\varepsilon)$ owing to an interference term between new physics and the SM contribution, whereas the third group has new physics contribution in the order of $\mathrm{O}\left(\varepsilon^{2}\right)$ in the rates, which is smaller. On the other hand, to identify new physics contribution, the SM contribution should be subtracted out for the second group, and theoretical uncertainty in the estimation of the SM contribution, which is sometime in the order of a few $\%$, would limit the sensitivity reach for new physics. And the sensitivity will improved roughly in $\sqrt{N}$ where $N$ is a number of total events. In the third group, subtraction of the SM contribution is not needed. The sensitivity will improve in a number of event $N$. From these considerations, the third group could be the best to search for new physics beyond the Standard Model in future, given the assumption that the number of $N$ can be significantly increased and the sensitivity can reach at the level of $\mathrm{O}\left(\varepsilon^{2}\right)$.

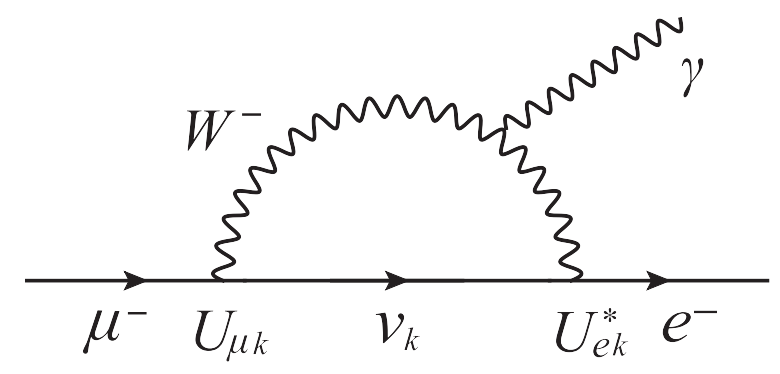

Figure 1: One of the diagrams of massive neutrino contributions to a $\mu$ to $e$ transition ( $\mu \rightarrow e$ “ $\gamma$ ").

One of the example processes where the SM contribution is forbidden is charged lepton flavor violating (CLFV) processes. For example, the prediction for $\mathrm{B}(\mu \rightarrow e \gamma)$ is shown in Fig. $\mathbb{\square}$, and 
given by,

$$
\begin{aligned}
\mathrm{B}(\mu \rightarrow e \gamma) & =\frac{\alpha}{2 \pi}\left|\sum_{k} U_{e k} U_{\mu k}^{*} \frac{m_{v_{k}}^{2}}{m_{W}^{2}}\right|^{2} \\
& \simeq \frac{\alpha}{2 \pi}\left|U_{e 3} U_{\mu 3}^{*} \frac{\Delta m_{\mathrm{atm}}^{2}}{m_{W}^{2}}\right|^{2}<10^{-54} .
\end{aligned}
$$

Here $U_{\beta i}$ is the Maki-Nakagawa-Sakata Matrix elements with $\beta$ denoting a charged lepton flavor eigenstate and $i$ a neutrino mass eigenstate with mass $m_{v_{i}}$, and $m_{W}$ is the $W$ boson mass, and $\alpha$ is the fine structure constant. Note that the GIM mechanism leads to a prediction dependent on differences in the masses of the neutrinos. For the $\mu^{+} \rightarrow e^{+} \gamma$ process, a similar suppression arises due to gauge symmetry.

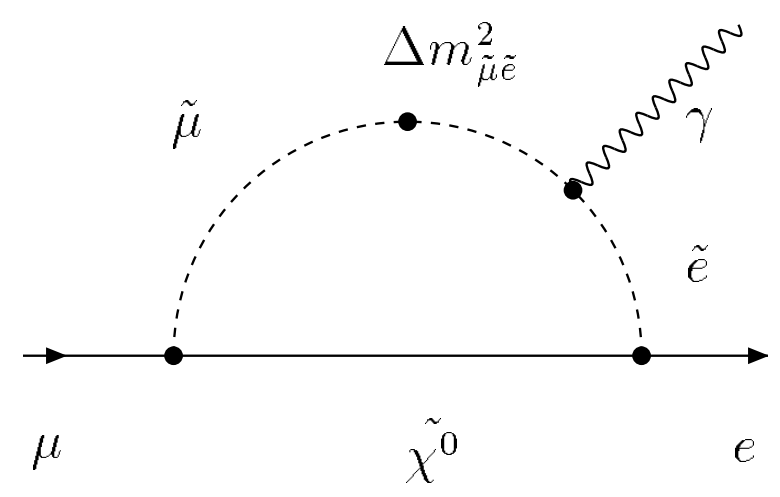

Figure 2: One of SUSY diagrams for CLFV

Searches for rare decays are sensitive to new physics beyond the SM. Various theoretical models predict sizable branching ratios for rare decays, being detectable in future experiments, if there are new particle at the energy scale not far from TeV. Let us take an example of CLFV. One of the physics models predicting sizable effects in CLFV is a supersymmetric (SUSY) model. In the SUSY models, CLFV occurs through the slepton mixing $\left(\Delta m_{\tilde{\mu} \tilde{e}}^{2}\right)$, as shown in Fig. \. It is given by an off-diagonal element of the slepton mass matrix. In SUSY-GUT models, $\left(\Delta m_{\tilde{\mu} \tilde{e}}^{2}\right)$ is proportional to $y_{t} V_{13} V_{23}^{*}$, and in the SUSY Seesaw models it is proportional to $y_{v_{3}} U_{13} U_{23}^{*}$, where $y_{t}$ and $y_{v_{3}}$ are the Yukawa couplings for the top quark and the neutrino $v_{3}$ respectively, and $V$ and $U$ are the CKM quark-mixing matrix and $P M N S$ neutrino-mixing matrix respectively. And for example, the branching ratio $(B)$ of $\mu^{+} \rightarrow e^{+} \gamma$ can be given by

$$
B \sim 10^{-13}\left(\frac{\tan \beta}{10}\right)^{2}\left(\frac{0.5 \mathrm{TeV}}{\tilde{m}}\right)^{4}\left(\frac{\Delta m_{\tilde{\mu} \tilde{e}}^{2}}{10^{-4}}\right)^{2} .
$$

It would produce sizable branching ratios. Therefore, if SUSY exits, the slepon mixing is sensitive to GUT (at $10^{16} \mathrm{GeV}$ ) or neutrino seesaw mechanism (at $10^{13-14} \mathrm{GeV}$ ). As a result, it can be concluded that CLFV has potential to study physics at very high energy scale. 


\section{Rare Muon Decays}

The most important rare muon decays are CLFV processes. There are two major muon CLFV processes, which are $\mu^{+} \rightarrow e^{+} \gamma$ decay, and $\mu^{-} \mathrm{N} \rightarrow e^{-} \mathrm{N}$ conversion.

For the search for $\mu^{+} \rightarrow e^{+} \gamma$ decay, the MEG experiment at Paul Sherrer Institute (PSI) is in operation. With their 2009 and 2010 data, they produced the upper limit of $\mathrm{B}(\mu \rightarrow e \gamma)<$ $2.4 \times 10^{-12}$ at $90 \%$ C.L. [四].The MEG experiment was running in 2012 , expecting a sensitivity of $O\left(10^{-13}\right)$ with the 2011 and 2012 combined data-sets. However the MEG sensitivity reach is slowing down due to backgrounds and detector resolutions. Therefore they are planning the detector upgrade to aim for a sensitivity of $O\left(10^{-14}\right)$ (MEG-II). Better detector resolution are critical for MEG-II so as to reduce accidental backgrounds when they use a 3 times higher beam intensity available at PSI. Possible detector upgrade would include, for instance, a single volume $e^{+} \mathrm{drift}$ chamber with all stereo wires, a Xe photon detector with MPPC readout with higher granularity, a silicon pixel timing counter, and an active target made of silicon or fibers, etc. The MEG-II is planning to start in 2015 or 2016 for a 3 year running period, after a long shut-down in 2013 and 2014.

Next experimental projects to search for $\mu^{-} \mathrm{N} \rightarrow e^{-} \mathrm{N}$ conversion with anticipated sensitivity improvement of four orders of magnitude are being pursued in the Fermi National Laboratory (FNAL), the USA and the the Japan Proton Accelerator Research Complex (J-PARC), Japan. In order to increase a muon beam intensity, a pion capture system where superconducting solenoid magnets of a high magnetic field surrounds a proton target to capture pions in a large solid angle. It leads a dramatic increase of muon yields by several orders of magnitude. And an experimental demonstration of the pion capture system to increase a muon production efficiency by a factor of 1000 has been made at the MuSIC facility, Osaka University [D]. At the same time, in order to suppress background events, in particular beam-related backgrounds, the following key elements have been adopted for the both experiments. They are, first of all, beam pulsing, which is required to eliminate beam-related backgrounds by performing measurements between beam pulses. To eliminate beam-related backgrounds from proton leakage, proton beam extinction is required during the measurement interval. Secondary. curved solenoids for muon transport are needed to select charges and momenta of muons as well as removing neutral particles in a beam. The principle is as follows. In a curved solenoidal magnetic field, a center of the helical trajectory of a charged particle is shifted perpendicular to the curved plane. The shift, whose amount is given as a function of momentum and its charge, makes a dispersive beam. By placing appropriate collimators, charges and momenta of muons can be selected. The muon transport solenoid system also maintains high transmission efficiency, resulting a significant increase of muon flux.

One proposal in the USA is the Mu2e experiment at FNAL. It was mostly based on the MELC design and aimed to search for $\mu^{-} \mathrm{N} \rightarrow e^{-} \mathrm{N}$ conversion at a sensitivity of better than $10^{-16}$. It consists of the production solenoid system, the transport solenoid system and the detector solenoid system. The Mu2e experiment is planned to combat beam-related background events with the help of a $8 \mathrm{GeV} / c$ proton beam of $7 \mathrm{~kW}$ in beam power from the Booster machine at FNAL. The Mu2e experiment at Fermilab is also aiming at a single event sensitivity of $\mathrm{B}(\mu+A l \rightarrow e+A l)<$ $3 \times 10^{-17}$. The Mu2e has passed DOE CD1, and plan to start in 2019 .

The COMET experiment in Japan is aiming at a single event sensitivity of $\mathrm{B}(\mu+A l \rightarrow e+$ 


\section{COMET Phase-I COMET Phase-II}
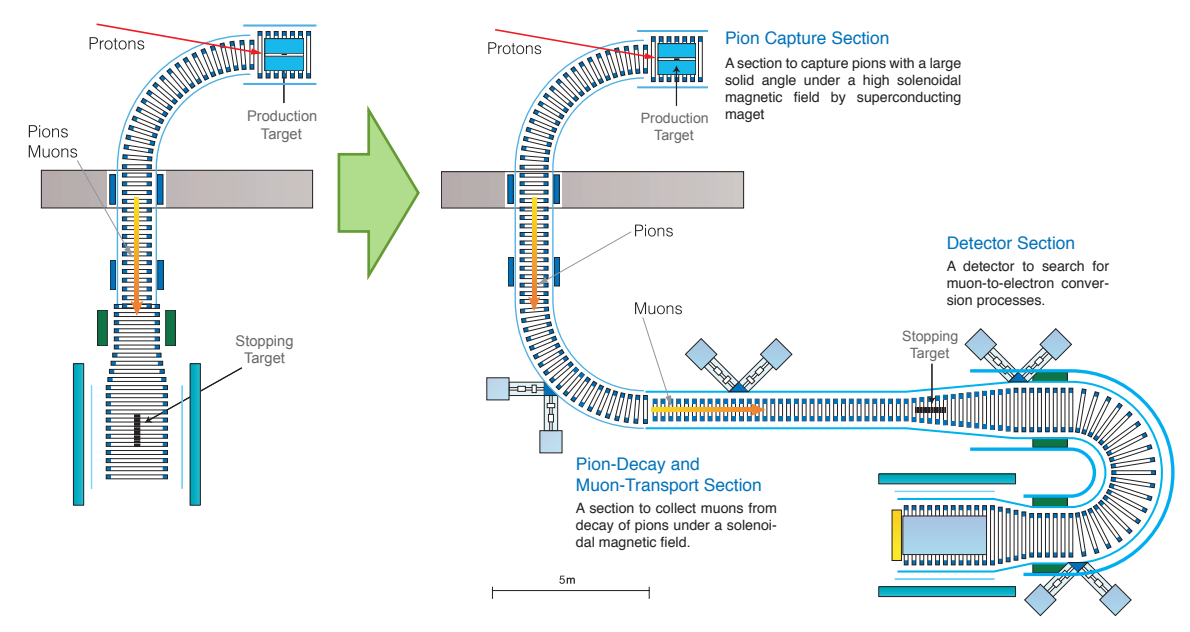

Figure 3: COMET staged approach: phase-I (left) and phase-II (right) [3]].

$A l)<3 \times 10^{-17}$ with about $10^{11}$ muons/sec [B] . The experiment was stage- 1 approved at J-PARC in 2009. The COMET experiment has adopted a staged approach to realize COMET in early time schedule, as shown in Fig. [3. The COMET staging scenario has been approved at the JPARC PAC and endorsed by the J-PARC review committee at MEXT in Japan. The COMET Phase-I would include the pion capture system and the muon transport system up to the end of the first 90 degree bend. The COMET Phase-I has two objectives, one of which is measurements of potential beam-related background sources, and the second is to search for $\mu-e$ conversion at an intermediate sensitivity, such as a single-event sensitivity of $3 \times 10^{-15}$, which is about a factor of 100 improvement over the previous with 0.03 background events. At the COMET Phase-I, a muon beam intensity of $5 \times 10^{9}$ muons/s with a $3 \mathrm{~kW}$ proton beam power is expected, and about $1.5 \times 10^{6} \mathrm{sec}$ running period (18 days) is sufficient to achieve an improvement of about 100 . KEK is planning to start the construction of the beam line in 2013. The COMET Phase-I will start in 2016.

\section{Rare Tau Decays}

Since a talk on CP violation, including tau leptons and light mesons was given in the ICHEP conference, in this ICHEP conference, CLFV decays with tau leptons is only mentioned as tau rare decays. The expected branching ratios for the CLFV processes with muons and taus are quite different for different theoretical models. It is worth to compare the results from tau leptons and muons to pin down the CLFV theoretical models.

The new report on upper limits on tau LFV decays from Belle was given [四]. The results are summarized in Fig. 田. The Belle collaboration is now working on the determination of new limits 


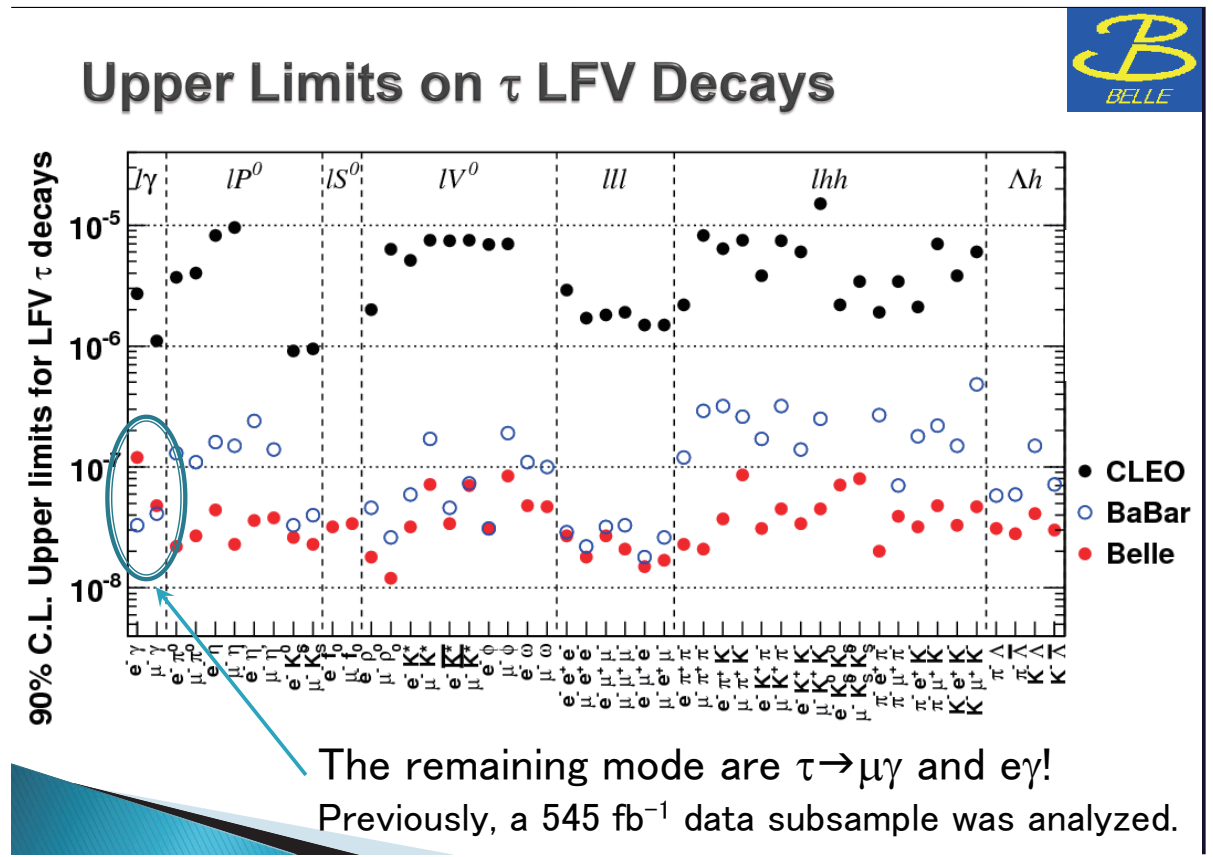

Figure 4: The upper limits of $\tau$ LFV decays obtained from Belle, reported by K. Hayasaka [四].

on $\tau \rightarrow \mu \gamma$ and $\tau \rightarrow e \gamma$ decays with the data of $980 \mathrm{fb}^{-1}$, which corresponds to about $10^{9}$ taus. The analysis is not complete yet, but limits of about $<5 \times 10^{-8}$ are expected.

A preliminary result on $\tau \rightarrow \mu \mu \mu$ decay of $\mathrm{B}\left(\tau^{-} \rightarrow \mu^{+} \mu^{-} \mu^{-}\right)<6.3 \times 10^{-8}$ at $90 \%$ C.L. from $\mathrm{LHCb}$ has been reported with the data of $1 \mathrm{fb}^{-1}$ [[]]. This new result is comparable with the Belle measurement of $\mathrm{B}\left(\tau^{-} \rightarrow \mu^{+} \mu^{-} \mu^{-}\right)<2.1 \times 10^{-8}$. An outlook at LHCb for 2012 is to accumulate and analyze data of $1.5 \mathrm{fb}^{-1}$.

\section{Rare Kaon Decays}

The lepton universality, in particular the $\mu-e$ universality, is one of the hot topics in rare $K$ decays. In the SM, the ratio $\left(R_{K}\right)$ of the decay width of $\Gamma\left(K^{+} \rightarrow e^{+} v\right)$ to $\Gamma\left(K^{+} \rightarrow \mu^{+} v\right)$ is given by

$$
\begin{aligned}
R_{\mathrm{K}}^{\mathrm{SM}} & =\frac{\Gamma\left(K^{+} \rightarrow e^{+} v\right)}{\Gamma\left(K^{+} \rightarrow \mu^{+} v\right)}=\left(\frac{m_{e}^{2}}{m_{\mu}^{2}}\right)^{2}\left(\frac{m_{K}^{2}-m_{e}^{2}}{m_{K}^{2}-m_{e}^{2}}\right)^{2}\left(1+\delta R_{K}^{r a d}\right) \\
& =(2.477 \pm 0.001) \times 10^{-5}
\end{aligned}
$$

where $m_{K}, m_{\mu}$ and $m_{e}$ are the masses of a kaon, a muon and an electron respectively. $\delta R_{K}^{\text {rad }}$ is the radiative correction. The hadronic contributions for the two decays are cancelled in the first order. In new physics beyond the SM, there are several theoretical extensions which predict sizable effects on $R_{K}$. One of them is the two Higgs doublet models, which have an extra charged Higgs particle $\left(H^{+}\right)$. It would introduce LFV at a one loop level. Similarly in minimum supersymmetric standard models (MSSM), it is expected that $R_{K}$ might have $1 \%$ effects [ [6]. In the Particle Data 
Group (PDG) $2010, R_{K}=2.493 \pm 0.0031(1.3 \%)$ is given. A new experiment to measure $R_{K}$ by using in-flight $K^{+}$decays at NA48/2 and NA62 of $\left.R_{K}=\left(2.488 \pm 0.007_{\text {stat }} \pm 0.007_{\text {sys }}\right) \times 10^{-5}\right)$ was reported in this conference [ $[\mathbf{}]$. It is still consistent with the SM value.

Rare kaon decays are also beneficial to study the Chiral Perturbation Theory (ChPT) at low energy. One of the rare kaon decays suitable to study ChPT is $K \rightarrow \pi \gamma \gamma$ decay. It would allow to study ChPT up to $O\left(p^{6}\right)$ where $p$ is momentum transfer. NA48/2 and NA62 reported the new measurement of $\mathrm{B}\left(K^{ \pm} \rightarrow \pi^{ \pm} \gamma \gamma\right)=(1.101 \pm 0.06) \times 10^{-4}$ [四]. It is also consistent with the PDG value of $\mathrm{B}\left(K^{ \pm} \rightarrow \pi^{ \pm} \gamma \gamma\right)=(1.10 \pm 0.32) \times 10^{-6}$.

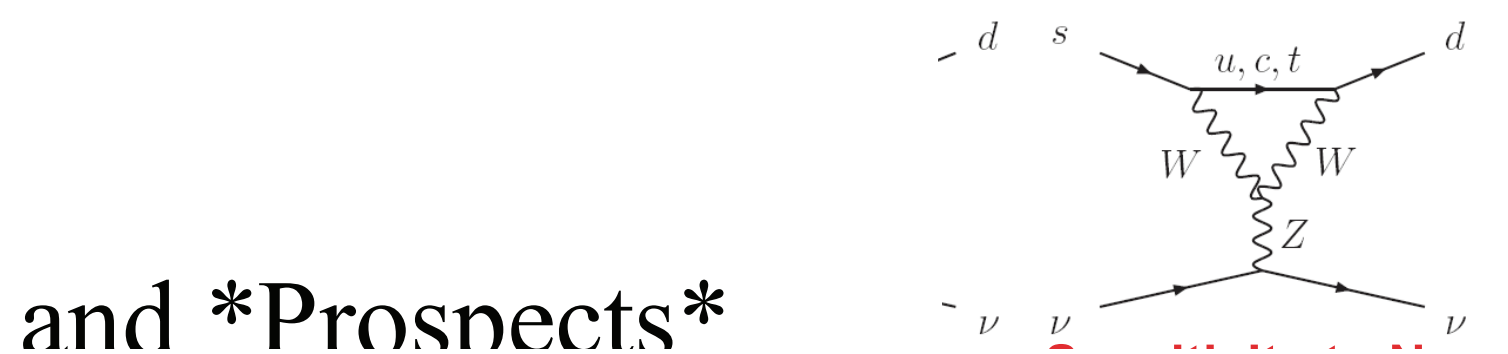

Figure 5: $W^{ \pm}$box diagrams and $Z$ penguin diagrams for $K \rightarrow \pi v \bar{v}$ decays

The golden modes of rare kaon decays are $K^{+} \rightarrow \pi^{+} v \bar{v}$ and $K_{L} \rightarrow \pi^{0} v \bar{v}$ decays. They are flavor-changing neutral current (FCNC) decays with $W^{ \pm}$box diagrams and $Z$ penguin diagrams, as shown in Fig. [1]. The SM predictions are given by $\mathrm{B}^{\mathrm{SM}}\left(K^{+} \rightarrow \pi^{+} v \bar{v}\right)=(7.2 \pm 0.8) \times 10^{-11}$ and $\mathrm{B}^{\mathrm{SM}}\left(K_{L} \rightarrow \pi^{0} v \bar{v}\right)=(2.8 \pm 0.4) \times 10^{-11}$. There are many expected new physics contributions, such as little Higgs models, Randall-Sundrum models and so on. In particular, by taking a ratio of the two, it would give better sensitivities to a search for new physics beyond the SM. The present measurement on $K^{+} \rightarrow \pi^{+} v \bar{v}$ is given by $\mathrm{B}\left(K^{+} \rightarrow \pi^{+} v \bar{v}\right)=\left(17.3_{-10.5}^{+11.5}\right) \times 10^{-11}$, based on 7 events measured by E787/E949 at Brookhaven National Laboratory (BNL).

The new experiment NA62 at CERN is being prepared to start data taking [D]. Their detector is shown in Fig 6 . They use in-flight $K+$ decays, aiming at 100 events with the uncertainty of $5 \%$. The timeline of NA62 is that they have the complete detector installation in 2013, and start datataking in 2014 with the full detector. The other future experimental proposal, ORKA, to measure $K^{+} \rightarrow \pi^{+} v \bar{v}$ at Fermilab was reported [ [ $]$ ]. The ORKA experiment would be the 4th generation detector, which will be using stopped $K^{+}$s. They are aiming about 1000 events collection in 5 years, yielding the uncertainty of $5 \%$ in the branching ration determination. Regarding $K_{L} \rightarrow \pi^{0} v \bar{v}$, the KOTO experiment at J-PARC has started data collection in 2012.

\section{Rare Charm Decays}

One of the important FCNC charm decays is $D^{0} \rightarrow \mu^{+} \mu^{-} . D^{0} \rightarrow \mu^{+} \mu^{-}$is highly suppressed in the SM. This SM contribution comes from long-distance contribution where two photon diagrams dominate, whereas the SM short distance contribution can be calculated to be much small, in the order of $\mathrm{O}\left(10^{-18}\right)$. The $\mathrm{SM}$ contribution of two photon diagrams is given by $\mathrm{B}\left(D^{0} \rightarrow \mu^{+} \mu^{-}\right) \sim$ 


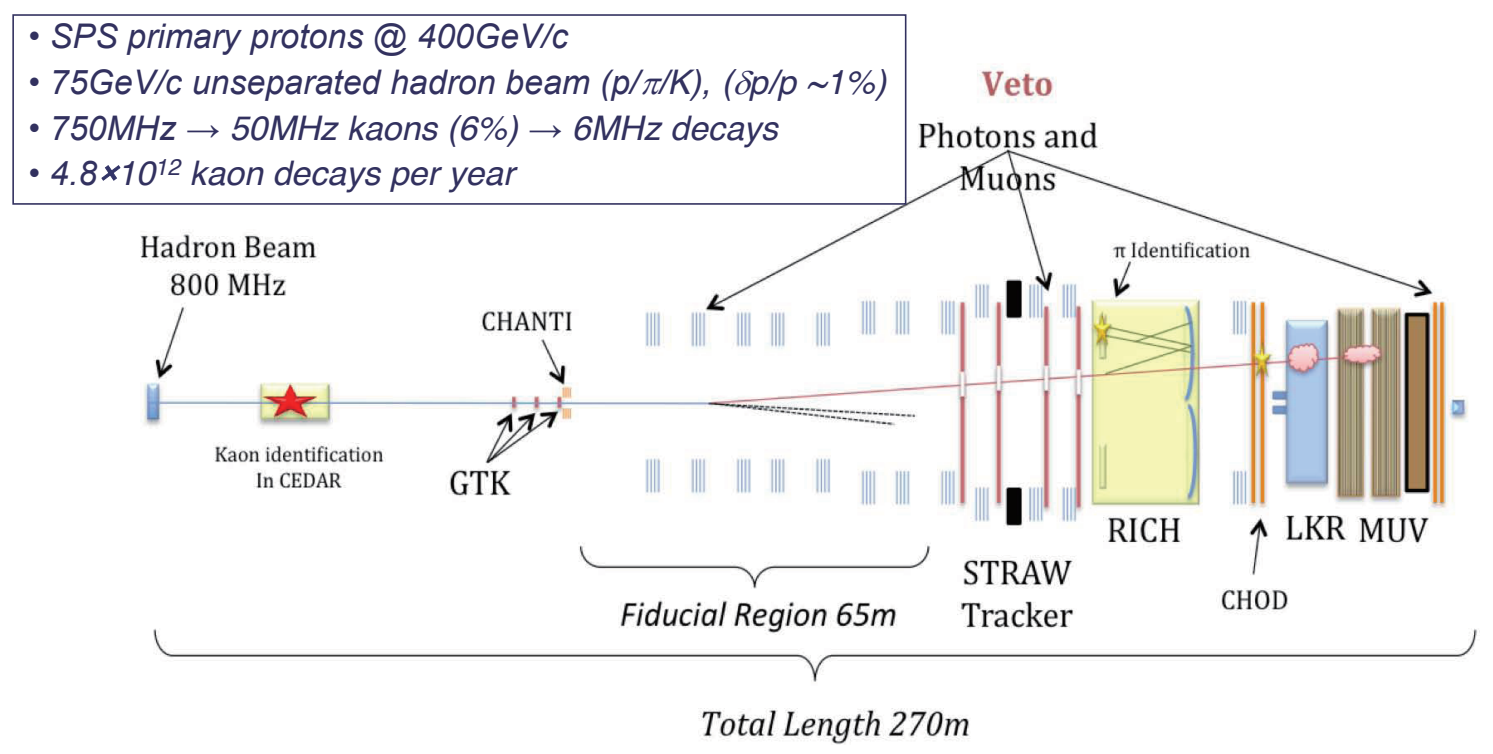

Figure 6: Schematic layout of the NA62 detector to measure $K^{+} \rightarrow \pi^{+} v \bar{v}[$ [ $]$.

$2.7 \times 10^{-5} \times \mathrm{B}\left(D^{0} \rightarrow \gamma \gamma\right)$. The $90 \%$ C.L. upper limit of $\mathrm{B}\left(D^{0} \rightarrow \gamma \gamma\right)<2.2 \times 10^{-6}$ was given by Babar, and therefore, $\mathrm{B}\left(D^{0} \rightarrow \mu^{+} \mu^{-}\right)<6 \times 10^{-11}$ at $90 \%$ C.L for the SM two photon diagram contribution can be obtained. Since it is very small, this decay mode is considered to be one of the best charm decays to search for new physics contribution. The current best limit on $\mathrm{B}\left(D^{0} \rightarrow \mu^{+} \mu^{-}\right.$. $\left.D^{0} \rightarrow \mu^{+} \mu^{-}\right)<1.4 \times 10^{-7}$ at $90 \%$ C.L. The new results on $\mathrm{B}\left(D^{0} \rightarrow \mu^{+} \mu-^{-}\right)$from $\mathrm{LHCb}$ and CMS were presented in this conference: the $90 \%$ C.L. upper limit of $\mathrm{B}\left(D^{0} \rightarrow \mu^{+} \mu^{-}\right)<1.1 \times 10^{-8}$ was obtained from $\mathrm{LHCb}$, and that of $\mathrm{B}\left(D^{0} \rightarrow \mu^{+} \mu^{-}\right)<5.4 \times 10^{-7}$ was obtained from CMS [Q] ]. On the other hand, Babar claimed $\mathrm{B}\left(D^{0} \rightarrow \mu^{+} \mu^{-}\right)=[0.6,8.1] \times 10^{-7}$ where 8 events were observed with expected backgrounds of $3.9 \pm 0.6$, although they set an upper limit on $\mathrm{B}\left(D^{0} \rightarrow e^{+} e^{-}\right)$. There is disagreements between the two sets of the results.

The Babar experiment also set the upper limits on CLFV decays of the D mesons. They are $\mathrm{B}\left(D^{0} \rightarrow e^{ \pm} \mu^{\mp}\right)<3.3 \times 10^{-7}$.

The leptonic decays of the $\mathrm{D}_{s}$ mesons are known to be sensitive to new physics beyond the SM. To search for new physics, the $\mathrm{D}_{S}$ meson decay constant, $f_{\mathrm{D}_{s}}$, has to be determined. The branching ratios of leptonic $D_{\mathrm{D}_{\mathrm{s}}}$ mesons are given by

$$
\mathrm{B}\left(D_{s}^{+} \rightarrow \ell^{+} v_{\ell}\right)=\frac{G_{F}^{2}}{8 \pi} f_{\mathrm{D}_{\mathrm{s}}}^{2}\left|V_{c s}\right|^{2} \tau_{D_{s}} M_{D_{s}} m_{\ell}^{2}\left(1-\frac{m_{\ell}^{2}}{M_{D_{s}}^{2}}\right)^{2}
$$

where $G_{F} . V_{c s}, \tau_{D_{s}} . M_{D_{s}} . m_{\ell}$ are the Fermi constant, the CKM matrix element, the lifetime of $D_{s}$, the mass of $D_{s}$ and the mass of lepton $\ell$ respectively. The Belle reported in this conference their preliminary result on $\mathrm{B}\left(D_{s}^{+} \rightarrow \mu^{+} v_{\mu}\right)=(0.528 \pm 0.028$ (stat.) $) \pm 0.019$ (syst.) $) \times 10^{-2}$, whereas the PDG value is $(0.590 \pm 0.033) \times 10^{-2}$ [ए0]]. The $D_{s}$ decay constant was obtained to be $257.2 \pm 4.5$ $\mathrm{MeV}$. The lattice QCD calculation gave $f_{D_{s}}=248.0 \pm 2.5 \mathrm{MeV}$. 


\section{Future Particle Accelerators and Facilities}

The rates of new physics contributions is given by

$$
R \propto \frac{1}{\Lambda^{4}},
$$

where $\Lambda$ is an energy scale of new physics beyond the SM. Therefore, if one aims to try to improve the $\Lambda$ reach by one order of magnitude, one must improve the sensitivity by $\mathrm{O}\left(10^{4}\right)$. resulting in the increase of the number of the parent particles in rare decays by $\mathrm{O}\left(10^{4}\right)$. To make significant progress on rare decay searches, highly intense accelerators are needed.

\subsection{High luminosity $e^{+} e^{-}$and proton colliders}

For high luminosity $e^{+} e^{-}$colluders, there are a fewl projects in the worldwide. One of them is the Super KEKB accelerator which is upgraded from the existing KEKB facility. It aims at 10 $\mathrm{ab}^{-1}$ by 2018 and $50 \mathrm{ab}^{-1}$ by 2022 . The second one is the Super-B factory in Italy, which aims at $75 \mathrm{ab}^{-1}$ after 5-year construction. ${ }^{1}$ In high luminosity $e^{+} e^{-}$colliders, CLFV in $\tau$ decays is one of the most important physics targets. Potential decay modes are $\tau \rightarrow \ell \gamma$ (where $\ell$ is either $e$ or $\mu), \tau \rightarrow \mu \mu \mu, \tau \rightarrow \mu \eta$ decays and so on. For $\tau \rightarrow \ell \gamma$ decay modes, the dominant background comes from $e^{+} e^{-} \rightarrow \tau^{+} \tau^{-}$with initial state radiation. It is not negligible and the upper limit can be proportional to $1 / \sqrt{N_{\tau}}$. With $50 \mathrm{ab}^{-1}$ of data, about $\tau$ pairs of $5 \times 10^{10}$ can be available. With the current signal-to-background ratio is maintained, an expected upper limit could be about $3 \times 10^{-9}$. For the other modes where backgrounds are negligible, the upper limit can be proportional to $1 / N_{\tau}$, yielding the upper limit of about $(0.2-1) \times 10^{-9}$.

For the LHC, the luminosity update was planned after the energy upgrade to $14 \mathrm{TeV}$. They planned to accumulate $1000 \mathrm{fb}^{-1}$, and then with the high luminosity update they will increase to $3000 \mathrm{fb}^{-1}$. The LHCb upgrade with $50 \mathrm{fb}^{-1}$ data set is also planned.

\subsection{Proton accelerators for muons and kaons}

Project-X in the USA is a high-power proton accelerator complex based on superconducting $\mathrm{RF}$ technology. It consists of $3 \mathrm{GeV}$ continues wave $(\mathrm{CW})$ proton linac, and a pulsed superconducting linac from $3 \mathrm{GeV}$ to $8 \mathrm{GeV}$, the existing $8 \mathrm{GeV}$ Booster and $120 \mathrm{GeV}$ Main Injector rings. It would provide ultimately beam powers of $3 \mathrm{MW}$ at $3 \mathrm{GeV}, 200 \mathrm{~kW}$ at $8 \mathrm{GeV}$ and $2 \mathrm{MW}$ at $120 \mathrm{GeV}$, simultaneously, and the $\mathrm{CW}$ beam can be configured as difference pulsed structure. One of the physics topics is rare muon decays. With multi MW beam power, Project-X could bring additional 100 times more muons of about $10^{13}$ muons/s.

Currently J-PARC is planning to achieve the design beam power of $750 \mathrm{~kW}$ in approximately five years. It could be achieved by increase of protons per bunch and high repetition rate of the J-PARC Main Ring (MR). At the same time, a study to realize a beam power in excess of $1 \mathrm{MW}$ is also underway. Some plans would include a rise of the injection energy to MR and others. Once a proton beam power above $1 \mathrm{MW}$ at J-PARC is available, variety of new flavor physics can be performed at J-PARC.

\footnotetext{
${ }^{1}$ Since the budget requested for Super-B factory was found to be incompatible with the available research funding, the project has been disconnected although the science case was strong enough.
} 
These future accelerators or the upgrade of the existing accelerators would provide beams of more intensity by $\mathrm{O}(10-100)$. It might not be enough to achieve $\mathrm{O}\left(10^{4}\right)$ increase in the number of particles, mentioned before. It would be desirable to consider how one can improve the efficiencies of particle production and particle collection, in addition to the primary beam intensity. The improvement of muon collection efficiency at the MuSIC facility at Osaka University was reported in this conference [ []]. In the MuSIC facility, a pion production target is surrounded by a high field superconducting solenoid magnet of $3 \mathrm{~T}$ so that most of pions can be captured by a solenoid magnetic field. A schematic layout of the MuSIC facility is shown in Fig $\square$. The basic idea is to have a long pion production target with a few interaction length, and collect pions emitted from the side of the pion production target by a solenoid magnetic field of a few T. At the MuSIC facility, an increase of the muon production yields by a factor of about 1000 has been observed at the MuSIC facility, compared with the current muon facilities. Together with highly intense proton machines, it would provide the increase of a number of muons by $\mathrm{O}\left(10^{4}\right)$.

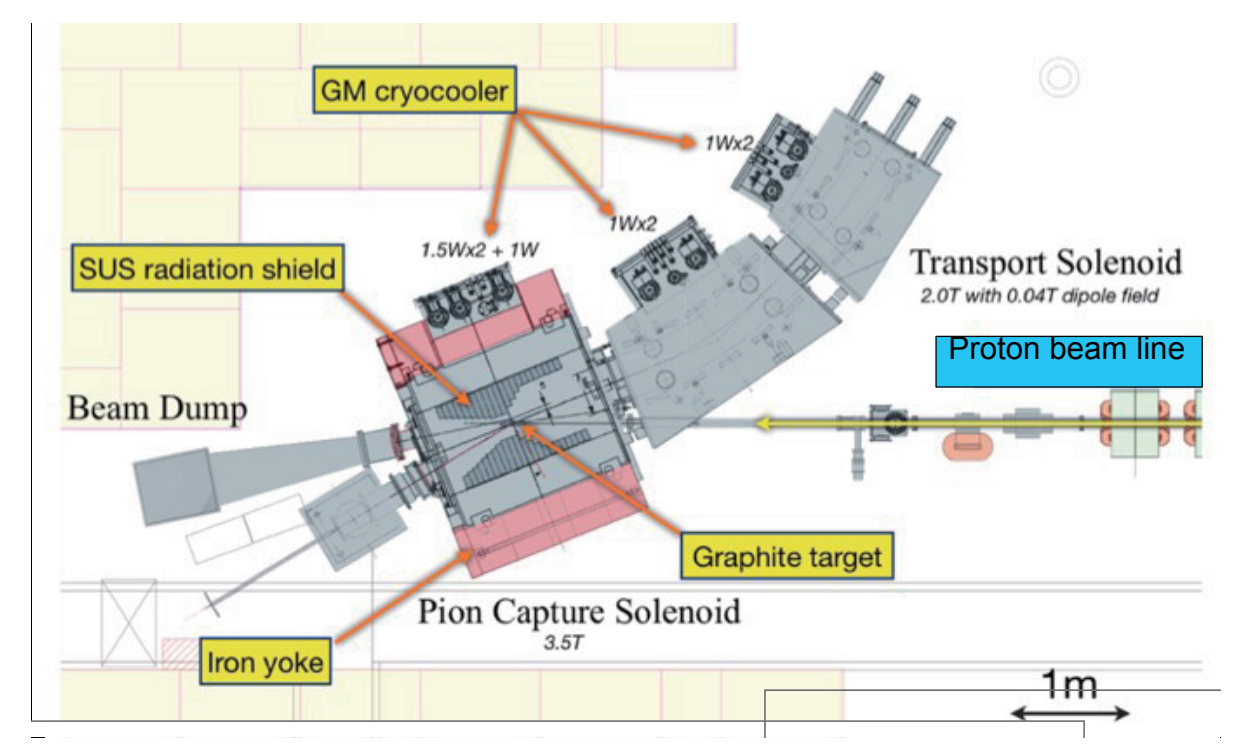

Figure 7: Schematic layout of the MuSIC muon source at Osaka University [Q]]. A proton beam comes from the right, and the muon beam is taken off to top.

\section{Summary}

We presented the experimental status of rare decays in charged leptons and light mesons. The physics of rare decays in charged leptons and light mesons, together is rich and is the most interesting in terms of searches for new physics beyond the SM at high energy scale.

\section{References}

[1] H. Nishiguchi's talk in this conference [829]

[2] Y. Hino's talk in this conference [634]. 
[3] Y. Kuno's poster presentation in this conference.

[4] K. Hayasaka' talk in this conference [742].

[5] M. Perrin-Terrin' talk in this conference [559].

[6] Massiro, Paradisi, Petronzio, Phys. Rev. D 74 (2006) 011701; JHEP 0811 (2008) 0421.

[7] V. Kekelidze's talk in this conference [152].

[8] M. Hildreth's talk in this conference [78].

[9] M. Bonivento's talk and K. Ulmer's talk in this conference [634].

[10] M.-Z. Wang's talk in this conference [718]. 